Weißhaupt, Mark; Hildebrandt, Elke; Hummel, Maria; Müller-Naendrup, Barbara; Panitz, Kathleen; Schneider, Ralf

\title{
Perspektiven auf das Forschen in Lernwerkstätten
}

Peschel, Markus [Hrsg.]; Kelkel, Mareike [Hrsg.]: Fachlichkeit in Lernwerkstätten. Kind und Sache in Lernwerkstätten. Bad Heilbrunn : Verlag Julius Klinkhardt 2018, S. 187-212. - (Lernen und Studieren in Lernwerkstätten)

Quellenangabe/ Reference:

Weißhaupt, Mark; Hildebrandt, Elke; Hummel, Maria; Müller-Naendrup, Barbara; Panitz, Kathleen; Schneider, Ralf: Perspektiven auf das Forschen in Lernwerkstätten - In: Peschel, Markus [Hrsg.]; Kelkel, Mareike [Hrsg.]: Fachlichkeit in Lernwerkstätten. Kind und Sache in Lernwerkstätten. Bad Heilbrunn : Verlag Julius Klinkhardt 2018, S. 187-212 - URN: urn:nbn:de:0111-pedocs-214289 - DOI: 10.25656/01:21428

https://nbn-resolving.org/urn:nbn:de:0111-pedocs-214289

https://doi.org/10.25656/01:21428

in Kooperation mit / in cooperation with:

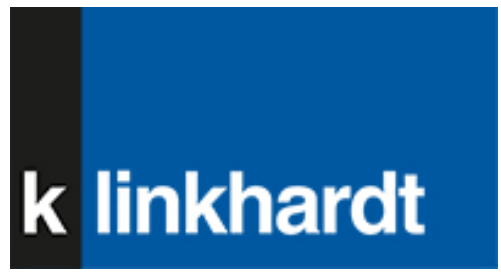

http://www.klinkhardt.de

\section{Nutzungsbedingungen}

Dieses Dokument steht unter folgender Creative Commons-Lizenz: $\mathrm{http}: / /$ creativecommons.org/licenses/by-nc-sa/4.0/deed.de - Sie dürfen das Werk bzw. den Inhalt unter folgenden Bedingungen vervielfältigen, verbreiten und öffentlich zugänglich machen sowie Abwandlungen und Bearbeitungen
des Werkes bzw. Inhaltes anfertigen: Sie müssen den Namen des Autors/Rechteinhabers in der von ihm festgelegten Weise nennen. Dieses Werk bzw. der Inhalt darf nicht für kommerzielle Zwecke verwendet werden. Die neu entstandenen Werke bzw. Inhalte dürfen nur unter Verwendung von Lizenzbedingungen weitergegeben werden, die mit denen dieses Lizenzvertrages identisch oder vergleichbar sind.

Mit der Verwendung dieses Dokuments erkennen Sie die Nutzungsbedingungen an.

\section{Terms of use}

This document is published under following Creative Commons-License: http://creativecommons.org/licenses/by-nc-sa/4.0/deed.en - You may copy, distribute and transmit, adapt or exhibit the work in the public and alter, transform or change this work as long as you attribute the work in the manner specified by the author or licensor. You are not allowed to make commercial use of the work. If you alter, transform, or change this work in any way, you may distribute the resulting work only under this or a comparable license.
mate

By using this particular document, you accept the above-stated conditions of use.

\section{Kontakt / Contact:}

\section{peDOCs}

DIPF | Leibniz-Institut für Bildungsforschung und Bildungsinformation

Informationszentrum (IZ) Bildung

E-Mail: pedocs@dipf.de

Internet: www.pedocs.de

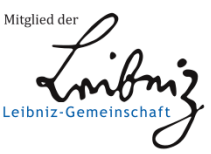




\section{Lernen und Studieren in Lernwerkstätten}

Markus Peschel

Mareike Kelkel

(Hrsg.)

Fachlichkeit in

Lernwerkstätten

Kind und Sache in Lernwerkstätten 


\section{Peschel / Kelkel \\ Fachlichkeit in Lernwerkstätten}




\section{Lernen und Studieren in Lernwerkstätten}

Impulse für Theorie und Praxis

Herausgegeben von

Johannes Gunzenreiner, Barbara Müller-Naendrup, Hartmut Wedekind, Markus Peschel und Eva-Kristina Franz 


\section{Markus Peschel \\ Mareike Kelkel \\ (Hrsg.)}

\section{Fachlichkeit in Lernwerkstätten}

Kind und Sache in Lernwerkstätten 
Der vorliegende Band ist aus der 9. Internationalen Fachtagung der Hochschullernwerkstätten hervorgegangen, die im Februar 2016 an der Universität des Saarlandes unter dem Thema: „Zur Sache! Fachbezüge in didaktischen Lernwerkstätten“ stattfand.

Dieser Titel wurde in das Programm des Verlages mittels eines Peer-Review-Verfahrens aufgenommen. Für weitere Informationen siehe www.klinkhardt.de.

Bibliografische Information der Deutschen Nationalbibliothek

Die Deutsche Nationalbibliothek verzeichnet diese Publikation in der Deutschen Nationalbibliografie; detaillierte bibliografische Daten sind im Internet abrufbar über http://dnb.d-nb.de.

2018.k. (C) by Julius Klinkhardt.

Das Werk ist einschließlich aller seiner Teile urheberrechtlich geschützt.

Jede Verwertung außerhalb der engen Grenzen des Urheberrechtsgesetzes ist ohne Zustimmung des Verlages unzulässig und strafbar. Das gilt insbesondere für Vervielfältigungen, Übersetzungen, Mikroverfilmungen und die Einspeicherung und Verarbeitung in elektronischen Systemen.

Coverfoto: CC0 Creative Commons/pixabay.de.

Druck und Bindung: AZ Druck und Datentechnik, Kempten.

Printed in Germany 2018.

Gedruckt auf chlorfrei gebleichtem alterungsbeständigem Papier.

ISBN 978-3-7815-2244-2

doi.org/10.35468/5652 


\section{Inhalt}

Markus Peschel und Mareike Kelkel

„Zur Sache!“

Mareike Kelkel und Markus Peschel

Fachlichkeit in Lernwerkstätten

Corinna Schmude und Hartmut Wedekind

Von der Sache aus denken und pädagogisch handeln.....

Sandra Tänzer und Elke Hohnstein

Das Lernen von Kindern in gemeinsamer Verantwortung begleiten

Pascal Kihm, Jenny Diener und Markus Peschel

Kinder forschen - Wege zur (gemeinsamen) Erkenntnis

\section{Linda Balzer}

Die Lernwerkstatt Religion Plural

Eva-Kristina Franz, Helga Huber, Vera Schauf und Sibylle Schwab

"Wer war denn nun eigentlich böse? Die Römer oder die Germanen?“

Anja Heinrich-Dönges, Holger Weitzel, Bernd Reinhoffer und Luitgard Manz

Forschend Sachunterricht studieren

Laura Dörrenbächer, Isabella Hart und Franziska Perels

Konzeption einer überfachlichen Lernwerkstatt für Lehramtsstudierende zur Förderung des selbstregulierten Lernens

Matthias Handschick, Lisa Stark, Eva Biard, Laura Delitala-Möller und Andreas Möller

Ästhetische Bildung im Spiegel von Lernwerkstattkonzepten:

Überlegungen zu interdisziplinären und übertragbaren Formaten der

Kulturvermittlung für heterogene Lerngruppen

Sabrina Schude

Die Entwicklung der Kasseler Lernwerkstätten und das Projekt

„Verzahnung der Studienwerkstätten“ 
Katrin Kaufmann, Franz-Josef Scharfenberg und Andrea Möller

Universitäre Lehr-Lern-Labore als multifunktionale didaktische

Lernwerkstätten

Mark Weißhaupt, Elke Hildebrandt, Maria Hummel,

Barbara Müller-Naendrup, Kathleen Panitz und Ralf Schneider

Perspektiven auf das Forschen in Lernwerkstätten

Barbara Holub

Lernwerkstatt als Herausforderung, Angebot und Chance

Ulrike Stadler-Altmann

EduSpaces - Räume für kooperativen Theorie-Praxis-Transfer

Verzeichnis der Autorinnen und Autoren 


\section{Mark Weißhaupt, Elke Hildebrandt, Maria Hummel, Barbara Müller-Naendrup, Kathleen Panitz und Ralf Schneider}

\section{Perspektiven auf das Forschen in Lernwerkstätten}

\section{Einleitung}

Die folgenden Überlegungen ${ }^{1}$ stellen die Frage ins Zentrum, in welchem Verhältnis „Forschungsprozesse“ von Kindern und das Erforschen kindlichen Lernens in Lern- und Forschungswerkstätten an Hochschulen stehen. Dazu werden theoretische Konzepte und Daten aus verschiedenen Lernwerkstätten präsentiert und folgende Fragen gemeinsam diskutiert:

- Welche(n) Forschungsbegriff(e) verwenden bzw. benötigen wir?

- Inwiefern gibt es Gleiches und Ungleiches beim Forschen, Spielen und Entdecken der Kinder und Studierenden?

- In welchem Verhältnis stehen kindliche zu wissenschaftlichen Forschungsprozessen und wo genau lassen sich Differenzen markieren?

- Wie können wir Studierende für Forschungsprozesse motivieren?

- Wie können wir Kinder in ihrem „Forschungsdrang“ unterstützen?

- Was sind dafür günstige Bedingungen, und welcher Typus von Begleitung stellt damit einhergehende handlungsleitende Kriterien zur Verfügung?

- Inwieweit ist die Verwendung des Forschungsbegriffs im Kontext kindlicher Entdeckungsprozesse angemessen, hilfreich, oder gar gefährlich?

- Wie kommen Kinder zur „Sache“, und wie können kindliche Lernprozesse unterstützt werden ohne dabei fachlich vorzeitig zu „disziplinieren“?

- Wie kann man im Rahmen von Lernwerkstattarbeit die Aufgaben der Lernbegleitung und die Erforschung kindlicher Lernprozesse ins Verhältnis setzen?

- Wie können Studierende dabei profitieren, „forschende“ Kinder in ihrem Lernen sachlich, fachlich und pädagogisch zu unterstützen bzw. dies in Praxis und in Auseinandersetzung mit Theorie forschend erlernen?

1 Ideen des Textes sind begründet in dem Theorie-Forum „Hochschullernwerkstätten als Forschungswerkstätten. Studierende erforschen, wie Kinder ,zur Sache' kommen“, das am 15.2.2016 in der Universität zu Saarbrücken unter Beteiligung von Annika Gruhn, Elke Hildebrandt, Maria Hummel, Barbara Müller-Naendrup, Kathleen Panitz, Ralf Schneider und Mark Weisshaupt stattfand. 


\section{„Verstehen kann nur jeder für sich selbst“ (Martin Wagenschein) - Forschung in und über Lernwerkstätten - Ein Resümee zu Beginn²}

Angesichts des umfangreichen Themenspektrums, das schon allein durch die in der Einleitung dieses Forum-Beitrags formulierten Diskussionsfragen eröffnet wird, ist es nicht einfach hier ein pointiertes Resümee aus der Sicht eines „critical friend" zu formulieren. Schnell wird deutlich, dass diese hier sowohl notwendige als auch durchaus berechtigte Diskussion um das Forschungspotenzial von Lernwerkstätten viele verschiedene Facetten hat, über die es sich sehr lohnt weiter nachzudenken.

Jedes der folgenden Kapitel greift für sich genommen wichtige unterschiedliche Perspektiven zum Thema „Forschung und Lernwerkstätten“ auf. Sie könnten durchaus auch als Einzelbeiträge veröffentlicht werden - doch das wollten ihre Urheber nicht -, denn es geht ihnen um eine vorsichtige Annäherung an ein sehr komplexes Thema in Verbindung mit Hochschullernwerkstätten. Im Folgenden versuche ich einen „roten Faden“ zu skizzieren:

- Es gilt als unbestritten, dass Lernwerkstätten an Hochschulen von ihrem konzeptionellen Selbstverständnis her nicht nur eine Basis, sondern vielmehr als „enabling spaces“ für forschende Lernprozesse, sowohl für Kinder als auch für Erwachsene verstanden werden können (Peschl \& Fundneider 2010, MüllerNaendrup 2012: 283; Kottmann 2013). Dies ist allerdings nicht als eine Art „Selbstläufer" zu betrachten, was im Übrigen für jeden Anspruch an Lernwerkstatt-Arbeit gilt (vgl. VeLW 2009), sondern es bedarf eines gründlich durchdachten und konstruktiven Zusammenspieles mehrerer Faktoren, wie z.B. ganz konkret durch Raum, Zeit, Ausstattung und im weiteren Sinne über die Einstellung aller Beteiligten und die Professionalität der jeweiligen Lernbegleiter (Kottmann 2013: 190; Gruhn \& Müller-Naendrup 2014).

- Hochschullernwerkstätten erheben insoweit berechtigt den Anspruch auch aber nicht nur - Forschungswerkstätten zu sein. Damit verstehen sie sich nicht als konkurrierende Einrichtungen zu anderen Forschungsstätten an Universitäten, denn sie sind sich ihrer Spezifität, begründet durch ihr Selbstverständnis, durchaus bewusst.

- Dem Anspruch einer Forschungswerkstatt nähern sich Lernwerkstätten in zweierlei Hinsicht: Zum einen bieten sie durch unterschiedliche Angebote und eine bewusst gestaltete „offene“ Lernumgebung einen sehr geeigneten Rahmen für forschende Lernprozesse sowohl für Erwachsene als auch für Kinder. Diese Lern- und Arbeitssituationen zeichnen sich durch einen hohen Grad an Selbständigkeit und partizipatorische Prozesse aus.

2 Kapitel von: Barbara Müller-Naendrup 
- Zum anderen werden unterschiedliche Aktivitäten und Angebote von Hochschullernwerkstätten nicht nur von VertreterInnen der Fachcommunity der Lernwerkstätten-Bewegung beforscht. Auch für externe Betrachter bieten diese Einrichtungen zunehmend ein interessantes Forschungsfeld.

- In der Lernwerkstatt SPIEL am Campus Brugg-Windisch führen Studierende eigene Forschungsprojekte zum Spiel von Kindern durch. In Kassel wird ein Projektseminar aus deutsch-didaktischer Perspektive beforscht. Zudem gibt es z.B. Forschungsprojekte, die nach der „Wirksamkeit“ von Lernwerkstatt-Angeboten fragen (vgl. Franz 2012) oder Projekte, die die forschenden Lernprozesse von Studierenden über qualitative Zugänge im Fokus haben (vgl. Gruhn 2017).

- Sowohl das konzeptionelle Selbstverständnis von Hochschullernwerkstätten als auch die realisierte Lernwerkstatt-Praxis vor Ort weisen von daher in vielerlei Hinsicht auf das Forschungspotenzial dieser Einrichtungen. Allerdings kennzeichnen die Beiträge auch die in der Lernwerkstatt-Idee begründeten Grenzen dieses Anspruchs. In der Lernwerkstatt Natur in Mühlheim erhalten Kinder von zwei bis sechs Jahren Gelegenheit, ihre eigenen Zugänge zu für sie bedeutsamen Phänomenen und Aspekten der Welt zu finden und zu erleben. Sie nehmen sich Zeit, erschließen sich den „Raum“ und bitten bei Bedarf auch um Hilfe für ihre eigenen Explorationen. Die Besonderheit dieser LernwerkstattSituation erinnert u.a. an ein Grundrecht des Kindseins, wie es der polnische Reformpädagoge Janusz Korczak mit dem „Recht des Kindes auf den heutigen Tag" fordert (Müller-Naendrup 2015: 33). Es geht um die Wertschätzung eines ,jeden Augenblicks“, der ganz dem Kind gehören sollte. Die ausgeprägte Neugier und das große Interesse von jungen Kindern, ihren eigenen Fragen nachzugehen, bieten sicherlich eine gute Basis für die Entwicklung einer forschenden Haltung in späteren Zusammenhängen. Vielfach wird beklagt, dass diese fruchtbaren Prozesse durch den „Abbaufaktor Schule“ unterbrochen werden, und es kaum gelingt, an späterer Stelle diese Kraft solcher explorativer Situationen erneut ins Leben zu rufen.

Hier greifen die „Machstrukturen schulischen Lernens“, wie sie Hagstedt (2014: 124) beschreibt: „Für die Kinder ist es nicht nur eine Enttäuschung, sondern auch eine Entwürdigung, an jedem Schulvormittag wieder neu zu erfahren, dass ihre eigenen Fragen für die Schule gerade nicht so wichtig sind wie für einen selbst, dass die Welt mit ihren vielen Geheimnissen ohnehin schon weitgehend entdeckt ist von den Erwachsenen, von Lehrplankommissionen und Lehrgangsentwicklern, von Schulbuchredakteuren und eben auch von ihren Lehrerinnen und Lehrern (die sich darauf berufen können)“.

- Lernwerkstätten könnten in diesem Zusammenhang durchaus als „Aufbaufaktor" eingeschätzt werden, da sie sowohl Kindern als auch Erwachsenen Gelegenheit, Raum und v.a. Zeit geben, ihren eigenen Fragen und Vorhaben vor Ort nachzugehen. Dabei gilt es zunächst den Eigenwert einer jeden Lern- und 
Arbeitssituation zu schätzen, ohne diese gleich mit der einseitigen „Forscherbrille" eines Erwachsenen zu deuten und gar zu lenken.

- Hierbei zeigen sich unterschiedliche rhetorische Strategien in Bezug auf Forschungsbegriffe und -bilder, wie z.B. dem des Experiments. Man kann den experimentellen Weltzugang als erwachsenenorientiertes Forschungsbild dem kindlichen Erfahrungslernen berechtigterweise entgegensetzen, um die kindliche Sphäre vor Zurichtung zu schützen (Lernwerkstatt Natur). Oder man kann gemeinsame Denkprozesse beim Experiment und bei der kindlichen Welterschließung herausarbeiten, um das spielerische Element in der Forschung (neu) zu beleben und um zu betonen, dass das explorative und quasi-experimentelle Denken von Kinderbeinen an bis zur (Hoch-)schule (neuen) Platz haben müsste (Lernwerkstatt SPIEL). Auch die Figur des reflected researcher (Grundschullernwerkstatt Kassel) stellt als solche zugleich eine Annäherung und eine Differenzierung von hochschulorientierten Forschungsbegriffen und der kindlichen Lernsituation dar. Ob und wie der als "didaktische Ethnograf“ beschriebene reflected researcher forschungsmethodische und didaktische Anforderungen ,unter einen Hut" bringen kann und sollte, halte ich für diskussionswürdig. Eine im pädagogischen Feld verankerte und zugleich reflexive Haltung empfiehlt sich aber in jedem Fall sowohl für den „Researcher" als auch für den didaktisch Tätigen (Gruhn \& Müller-Naendrup 2016).

- Eine weitere Grenze der möglichen Forschungsaktivitäten im Kontext von Hochschullernwerkstätten ist sicherlich durch die Passung der ausgewählten Forschungszugänge und -methoden mit dem zugrundeliegenden Selbstverständnis dieser Einrichtungen gegeben. Auf dem jetzigen Stand sind hier meiner Meinung nach qualitative Zugänge zu bevorzugen, da sie dem Wert der einzelnen Situation am ehesten gerecht werden. Methoden der Ethnographie bieten sich, je nach Vorhaben, gleichermaßen an, wie u.a. Projekte im Sinne der Aktions- und Praxisforschung. Wichtig dabei ist, dass im betreffenden Diskurs eine klare und präzise fachliche $\mathrm{Zu}$ - und Einordnung erfolgt.

Die hier beschriebenen Wege, das Feld zwischen kindlicher Exploration und (erwachsener) Forschung differenzierend, vergleichend und im Hinblick auf adäquate Begleitung in den Blick zu nehmen, bieten vielfältige Anschlussmöglichkeiten für Theorie und Praxis der Forschung in Hochschullernwerkstätten und des kindlichen Lernens. „Verstehen kann nur jeder für sich selbst“: Diesen Grundsatz von Martin Wagenschein (Buck 2012), zu deuten vor dem Hintergrund des genetischen Prinzips, kennzeichnet meiner Meinung nach deutlich das Potenzial, die Vielfalt und auch die Grenzen von Forschung in und über Lernwerkstätten. Das folgende Theorieforum zu diesem Thema bietet eine wichtige und gehaltvolle Basis für einen Diskurs, der gegenwärtige sowie zukünftige Projekte professionell initiiert, begleitet und perspektivisch analysiert. 


\section{Wann ist es Forschung? Zu Merkmalen und Begleitung von spielerischem und forschendem Lernen bei Kindern und Studierenden ${ }^{3}$}

Um uns den vorliegenden Fragen zum Erforschen des kindlichen „Forschens“ zu nähern, knüpfen wir zunächst an den Diskurs darüber an, ob es einen Unterschied zwischen Lernwerkstatt und Forschungswerkstatt gibt, und wenn ja, worin sich dieser Unterschied ausdrückt. Ralf Schneider und Theresa Schwarzkopf geben zu dieser Frage des Unterschieds die Antwort, dass es sich um zwei unterschiedlich ausgeprägte Formen des Lernens handelt, einerseits des entdeckenden und andererseits des forschenden Lernens, die in den zwei unterschiedlichen WerkstattFormaten beheimatet sind, dort gepflegt und inspiriert werden (Schneider \& Schwarzkopf 2013: 175ff, vgl. Abb. 1).

\begin{tabular}{|c|c|c|}
\hline Kategorie & Lernwerkstatt & Forschungswerkstatt \\
\hline $\begin{array}{l}\text { Grundlegendes } \\
\text { Lernkonzept }\end{array}$ & Entdeckendes Lernen & Forschendes Lernen \\
\hline Raum und Material & Am Material lernen & $\begin{array}{l}\text { Interesse und Frage aus sich selbst } \\
\text { entwickeln }\end{array}$ \\
\hline Gegenübers & $\begin{array}{l}\text { Es kann am und mit einem } \\
\text { Gegenüber gelernt werden, muss } \\
\text { aber nicht }\end{array}$ & $\begin{array}{l}\text { Ein Gegenüber ist für die } \\
\text { (Meta)Kommunikation zwingend } \\
\text { (Materialersatz) }\end{array}$ \\
\hline Rolle des Lehrenden & $\begin{array}{l}\text { Lernbegleiter und Arrangeur von } \\
\text { Lernumgebung }\end{array}$ & $\begin{array}{l}\text { Lernbegleiter und Moderator für } \\
\text { Arbeitsgruppen/Lernsituationen }\end{array}$ \\
\hline $\begin{array}{l}\text { Lerngegenstände/ } \\
\text { Forschungsgegenstände }\end{array}$ & Keine Einschränkung & Aus dem berufspraktischen Feld \\
\hline
\end{tabular}

Abb.1: „Ausschnitt aus dem Beratungsleitfaden der Forschungswerkstatt FLEx“ (Schneider, R. \& Schwarzkopf, T. 2013: 179).

Die stark material-basierte, im Umgang mit Material „entdeckte“ und thematisch freie Ausgangsfrage von lernenden Kindern und auch Studierenden in Lernwerkstätten steht hier einem forschenden Lernen gegenüber, bei dem die Forschungsfrage maßgeblich in bereits bestehenden, inneren Interessen des Forschenden begründet ist. Beim forschenden Lernen wird demnach die Forschungsfrage vor allem im Dialog, u.a. mit der Lernbegleitung, entwickelt, wobei Material bei der Entwicklung dieser Forschungsfrage nicht zentral, in manchen Fällen sogar hinderlich sein kann und insofern eine gewisse Nüchternheit der Werkstatt sinnvoll ist (ebd.: 177).

Letzteres steht in einem starken Kontrast zur in der Lernwerkstätten-Arbeit traditionell verbreiteten Haltung, durch reichhaltiges Material und dessen „Entde-

3 Kapitel von: Mark Weißhaupt und Elke Hildebrandt 
ckung" könnten nachhaltige Forschungsprozesse initiiert werden, wobei oft nicht zwischen forschenden und entdeckenden Aktivitäten deutlich unterschieden wird: „[...] die Beschreibung eines Ortes [...], an dem vorrangig Erwachsene [...] aktiv, forschend, entdeckend, kreativ und offen wie Kinder lernen sollten" (VeLW 2009: 5).

Hier geht es nicht zuletzt um die Frage nach der „Dignität“ der jeweiligen Konzepte. Man könnte überspitzen: einerseits der reformpädagogische didaktische Optimismus der Pädagogik Entdeckenden Lernens der Lernwerkstätten, andererseits die Tendenz der Verwissenschaftlichung der Lernwerkstatt-Mathetik durch eine Differenzierung des Forschungsbegriffs vom Entdeckenden Lernen. Diese Argumentation wurde von Hildebrandt et al. (2014) am Vergleich traditioneller Lernwerkstätten mit Forschungswerkstätten mit folgenden Schlüssen diskutiert: Nehmen einerseits angehende Lehrpersonen exemplarisch an Studien teil, dient dieses Forschende Lernen (Schneider \& Wildt 2002) ${ }^{4}$ dem Aufbau theoretischen Wissens und der Reflexionskompetenz, was wiederum die Qualität von Praxisreflexion erhöhen kann. „Beide Werkstattkonzeptionen haben also ihren je eigenen Wert: Lernen über reflektierte Praxis und Forschendes Lernen anhand von Teilnahme an Studien“ (ebd.: 96). Lern- und Forschungswerkstatt gemeinsam wäre wiederum der ureigene Ansatz von Lernwerkstätten, dass Studierende dort selbstständig arbeiten und eigenen Themen nachgehen.

Man könnte die Forschungsaktivitäten im Bereich Lernwerkstätten auch nach der formalen Einbettung unterscheiden:

1. Studierende nehmen an Studien teil bzw. führen sie durch.

2. Kinder, Studierende oder Lehrpersonen finden ihre Forschungsfrage bzw. ihr Projektziel und werden dabei von Lernbegleitern und Material unterstützt.

Die wissenschaftstheoretisch angezielte Offenheit der Forschung und ihrer Ergebnisse (Beywl et al. 2015; Popper 2009) steht oftmals in einem irritierenden Kontrast zur vielfach tatsächlich anzutreffenden Faktenvermittlung an den Schulen (Hildebrandt \& Weißhaupt 2018 i.V.). Sowohl in humanistischer Bildungsphilosophie humboldtscher Prägung (Humboldt 1809/1934) als auch in den Fundamenten empirischer Wissenschaft, wie dem kritischen Rationalismus, ist immer wieder hervorgehoben worden, Erkenntnis als etwas Vorläufiges zu beschreiben (Popper 2009): bei Humboldt als „unaufgelöste Probleme“, die im Kontext von Wissenschaft bearbeitet werden, bei Popper als zentrale methodologische Annah-

4 „Forschendes Lernen im Kontext schulpraktischer Studien bezeichnet einen wissenschaftsgeprägten Zugang zur pädagogischen Berufspraxis. In Anknüpfung an Methoden entdeckenden und projektorientierten Lehrens bzw. Lernens integriert dieser Zugang Forschungsstrategien der Humanund Sozialwissenschaften in das hochschuldidaktische Konzept zur Gestaltung von Praxisstudien“" (Schneider \& Wildt 2002: 10). 
me bei der Überprüfung bzw. Falsifikation von Hypothesen, während es in der Schule vor allem um wissenschaftlich geprüfte Wissensbestände geht.

Schule stellt sich dabei in vielerlei Hinsicht als Abbaufaktor für kindliche Neugier und Wissensdurst dar (Angelone et al. 2013), insbesondere wenn dort der Eindruck erweckt wird, als seien wissenschaftliche Erkenntnisse etwas Abgeschlossenes bzw. endgültig Faktisches. Dabei sind und bleiben sie etwas stets weiter Befragbares, zur Weiterbeforschung Herausforderndes. Da in der Schule die Wissenschaftlichkeit des Wissens ganz zentral im Mittelpunkt stehen sollte (Oelkers 1997, Morris et al. 2012), ist zu fragen, ob (nach)entdeckendes Lernen in Lernwerkstätten der Wissenschaft nicht nähersteht als der schulischen Praxis. Um dieses Verhältnis genauer zu untersuchen, stellt sich die Frage, inwiefern es Gemeinsamkeiten und Differenzen zwischen forschendem und entdeckendem Lernen gibt. Wedekind und Schmude (2017) beantworten diese Frage mit der Betonung der Differenzen der beiden Lehr-Lern-Formate. Danach geschieht entdeckendes Lernen in Lernwerkstätten mit der Zielrichtung der individuellen Erfahrung in der Begegnung mit Gegenständen. Hierbei seien „didaktische Implikationen für die eigene pädagogische Arbeit abzuleiten“ (ebd.: 199). In Forschungswerkstätten gehe es vor allem darum „das Forschen zu lernen“ (ebd.), wobei beide LehrLern-Formate zur Ausbildung pädagogischer Handlungskompetenz notwendig seien. Insoweit lässt sich in der jüngeren Diskussion eine relativ große Übereinstimmung bei der Differenzierung von forschendem und entdeckendem Lernen feststellen (Wedekind \& Schmude 2017; Hildebrandt et al. 2014; Schneider \& Schwarzkopf 2013; Schneider \& Wildt 2002).

Um die Gegenüberstellung von forschendem und entdeckendem Lernen speziell zu beleuchten, und dabei gerade auch die Übergänge neben den Differenzen herauszuarbeiten, sollen im Folgenden Prozesse im Spiel betrachtet werden, die die Art und Weise des Entdeckens bei Kindern genauer konturieren. Dabei soll das Verhältnis zum forschenden Lernen neu beschrieben werden: nicht vor allem in einem Gegensatz, sondern in einem sich gegenseitig fördernden Verhältnis (Hildebrandt \& Weißhaupt 2018 i.V.).

Es stellt sich in diesem Zusammenhang zunächst die Frage: Was ist Forschung? Was meinen wir genau, wenn wir z.B. sagen „Kinder forschen“? Müssen sich die Kinder dabei bewusst sein, „Forschung“ zu betreiben? Und was heißt das genau: sich dessen bewusst sein, Forschung zu betreiben?

Die Frage bezieht sich auch auf die zeitliche und methodische bzw. methodologische Struktur: Muss man, um Forschung im wissenschaftlichen Sinne zu betreiben, explizit unterscheiden zwischen Fragestellung, Hypothese, Operationalisierung, Datenerhebung, methodische Datenauswertung, Schlussfolgerung? Muss man diese Phasen zeitlich auseinanderziehen, und muss man dies begrifflich fassen können, um „zu forschen“? Was ist begleitendes unterstützendes Handeln 
bei der Forschung? Was ist der Gegensatz zur Begleitung von Spiel? Und die Gegenfrage: Wodurch zeichnet sich Spiel aus?

Betrachten wir zunächst das Konstruktionsspiel: Über die praktische und spielerische Auseinandersetzung mit Materialien werden Kinder zu experimentellen Handlungs- und Denkprozessen angeregt (Hauser 2013). Im Verlauf stellen sich Fragen wie: Klappt der Zusammenbau der Einzelteile und funktioniert das fertige Werk wie gedacht? Oder bricht die Konstruktion zusammen? Wie könnte man es anders bauen? In diesem Handlungs- und Spielprozess haben die Kinder Modelle im Kopf (Wygotski 1980) und experimentieren aufgrund dieser mit Materialien, passen ihre Modelle den Ergebnissen, Erfolgen und Misserfolgen an und verbessern sie dabei immer wieder. So wird im Konstruktionsspiel zeitlich zusammengezogen und impliziert: Modellentwurf, Versuch, Korrektur, neues Konstrukt/Modell, Weiterentwicklung der Konstruktion/des Modells. Zudem setzen sich Kinder dabei auf einer handelnden Ebene auch mit Modellen physikalischer Gesetzmäßigkeiten auseinander, oft ohne sie im Vorhinein zu kennen, explizit zu nennen oder im Detail formulieren zu können (Kraft, Gravitation, Balance, Reibung, Trägheit, Fliehkräfte usw.). Mit anregenden Impulsen verbaler und nonverbaler Art und Herausforderungen für weitere Aktivitäten und Verbalisierungen werden solche Prozesse unterstützt (Kucharz et al. 2014: 72). Dies kann auch schon in relativ kurzen Spiel-Lernphasen geschehen, indem die Kinder z.B. angeregt werden, über ihre mentalen Konzepte zu sprechen und diese so innerhalb des Spiels weiterentwickeln zu können.

Es stellt sich die Frage: Sind dies bereits „forschende Prozesse“, „forschendes Lernen“ (Huber et al. 2009, Schneider 2009, Kottmann 2013) oder „Forschungsprozesse“? Wir würden argumentieren: Die genannten Spielprozesse sind noch nicht forschende Prozesse im strengen Sinne - und das ist auch gar nicht nötig oder wünschenswert (siehe dazu auch im nachfolgenden Kapitel „Explorative vs. forschende Denkbewegungen von Kindern am Beispiel der „Lernwerkstatt Natur") - sie bereiten u.a. Forschung vor, sie üben grundlegende Denkwerkzeuge des Forschens ein, sie können manchmal(!) zur Forschung übergehen - im Spiel intrinsisch motiviert -, ohne dass man dies als Selbstzweck forcieren müsste. Dies geschieht über eine im Prozess selbst gewählte oder im Dialog entstandene, aber in jedem Fall vom Kind proaktiv angenommene Herausforderung oder über die Vorstellung eines gewünschten Ziels bzw. des nächsten Schrittes im Spiel (Oerter 1999), der zu Handlungsentwürfen herausfordert. Der Weg zu einem - entstandenen und generell noch wandelbaren - Ziel kann dabei offen bleiben. Im Spielfluss wird dieser entdeckt und nicht kleinschrittig von außen vorgegeben.

Wenden wir uns nun einer anderen Spielform zu, die vielleicht weniger deutlich von entdeckenden in forschende Prozesse überzugehen scheint, dem Rollenspiel: Was hat das Rollenspiel mit forschenden Prozessen zu tun? 
Es scheint auf der einen Seite das ernste und methodische Streben nach Erkenntnis und auf der anderen Seite ein kontrafaktisches, spielerisches „So-tun-als-ob“ zu stehen. Aber genau darin ist auch die enge Verbindung begründet: Das kontrafaktische Denken ist ein Denken in vorläufigen Modellen.

So werden im Rollenspiel nach Andresen (2002; 2003)

- Kontexte konstituiert,

- nonverbale und vor allem sprachliche Aushandlungsprozesse vollzogen,

- Objekte umgedeutet, de- und rekontextualisiert, mit ihnen interagiert,

- kontrafaktische Rollen und Skripts definiert und verhandelt,

- Geltungsansprüche vertreten,

- Meta-Kommunikationen und zunehmend abstraktere Denkprozesse vollzogen. So wird der Sinn für spezifische Zugangsweisen zur Wirklichkeit geschärft und komplexes, abstraktes Denken eingeübt (Wygotski 1980; Weißhaupt \& Campana 2014; Hildebrandt, Güvenç \& Pautasso 2015).

Es gilt also für beide hier aufgerufenen Spielformen Konstruktionsspiel und Rollenspiel: Kinder entwickeln mentale Modelle für ihr Spiel und testen sie interaktiv aus, mit Objekten und nicht zuletzt im Dialog mit den versprachlichten Modellen der jeweils anderen Mitspielenden. Dies hängt eng mit dem Begriff Spiel und seiner Verbindung zum Lernen und zur Bildung zusammen: Die spielenden Kinder sind sich ihres Anteils an der Konstruktion der Welt im Spiel bewusst (Jörrisen 2009; Weißhaupt \& Campana 2014). Das Denken in vorläufigen Modellen, die sich im Prozess des Spielens bewähren, fallen gelassen oder weiterentwickelt werden, wird beim Spiel (neben anderen Dingen) eingeübt und beim Rollenspiel auch metasprachlich angezeigt und bearbeitet (Giffin 1984; Bose 2003; Andresen 2002, 2011; Hildebrandt, Güvenç \& Pautasso 2016). Man kann das Spiel an dieser Stelle als wissenschaftspropädeutische Sphäre kennzeichnen.

Spielbegleitung bzw. pädagogisches Handeln im Bereich Spiel besteht entsprechend u.a. in der Fähigkeit, die Balance zu halten bzw. wechseln zu können zwischen der Spielwelt und der Außenwelt, und dabei das Spiel und seine impliziten wie expliziten Verhandlungen von Modellen, Konzepten und Ideen unterstützen oder sogar anreichern zu können, ohne die eigene Dignität und den „heiligen Ernst" des Spiels dabei zu gefährden (Heimlich 2015, Huizinga 2011, Weißhaupt \& Campana 2014). Anstatt die spielerische Konstruktion der Welt der Kinder mit Instruktion von außen über die erwachsenen, angezielten oder schulstoffentsprechenden und deshalb vermeintlich „abgesicherten“ Konstruktionen abzubrechen und sich dabei als „Spielverderber“ zu betätigen, müsste als gemeinsames Merkmal Spiel-, Lern- und auch Forschungsbegleitung die Vorläufigkeit der Modelle und Konstruktionen mitkommunizieren - die Begleitung müsste mitspielen können. Stattdessen hemmt Schule viel zu oft die kindliche Lust an der Exploration, der Welterschaffung und -erschließung (Angelone, Keller \& Moser 2013). 
Während zuvor die Bedeutung von Spielprozessen für die Entwicklung einer forschenden Haltung diskutiert wurde, fragen wir nun nach der Forschung zum Spiel. In der Lernwerkstatt SPIEL werden die Studierenden animiert, zum Spiel von Kindern eigene kleine Forschungsprojekte durchzuführen (Hildebrandt \& Weißhaupt 2018 i.V.). Da die Studierenden im wissenschaftlichen Sinne forschende Novizen sind, auch wenn sie im Rahmen von drei Seminaren an der Pädagogischen Hochschule in der Nordwestschweiz auf wissenschaftliches Arbeiten vorbereitet werden, ist die Perspektive zunächst auf das Entwickeln des Forschenden Lernens gerichtet. Gerade hier stellt sich die Herausforderung, die eigenen Forschungsinteressen der Studierenden mit den wissenschaftlichen Spielregeln im Verlauf zu verknüpfen, insbesondere um den lernenden- und studierendenzentrierten Fragen und Problemen (Hagstedt \& Krauth 2014: 17) einen nachhaltigen Platz in der Hochschule zu sichern.

In Seminaren und im freien Zugang zur „Lernwerkstatt SPIEL“ am Campus Brugg-Windisch (Schweiz) dürfen und sollen die Studierenden durchaus auch entdeckend lernen, vom Material ausgehend, spielend und erfahrungsbasiert (Schäfer \& Hauser 2014), einerseits um zum Thema Spiel in direkter Erfahrung (zurück) zu kommen, nicht zuletzt aber auch um die spielerische, in Modellen denkende Haltung in Wissenschaft wieder entdecken zu können, und so die Verknüpfung von entdeckendem und forschendem Lernen selbst herzustellen.

Studierende haben hier nicht zuletzt Teil an wissenschaftlichen Studien. So besuchten in einer Studie zum Rollenspiel Studierende eines Seminars lokale Kindergärten und erhoben Daten zu Spielprozessen beim Rollenspiel (Hildebrandt, Güvenç \& Pautasso 2015; 2016). Es wurden anhand von Audio- und Videodateien Transkriptionen von Peer-Interaktionen sowie von Interaktionen der spielenden Kinder und der Lehrperson angefertigt. Diese interpretierten die Studierenden nach methodischen Vorgaben in Gruppen, gerade auch mit dem Ziel, die Schärfung des genauen, forschenden Blicks habituell einzuüben. Die Lehramtsstudierenden setzen sich parallel zu den inhaltlichen Erkenntnissen zur Bildung im Spiel sowohl mit ihrer eigenen Haltungsentwicklung als auch dem Erwerb praktischer Fertigkeiten auseinander. Dabei wenden sie (Spiel-)Regeln der Forschung an, welche die Konstruktion des jeweils aktuellen, vorläufigen Wissensstandes und die Weiterentwicklung von Modellen betreffen, d.h. auch, dass sie (wieder) erlernen in vorläufigen Modellen zu denken. Tabelle 1 gibt einen Überblick darüber, wie sich Spiel als wissenschaftspropädeutische Aktivität und Forschung zueinander verhalten. 
Tab. 1: Differenzen zwischen Spiel- und Forschungsprozessen

\begin{tabular}{|c|c|c|}
\hline $\begin{array}{l}\text { Forschungs- und } \\
\text { entdeckungsrele- } \\
\text { vante Aspekte }\end{array}$ & $\begin{array}{l}\text { Spiel als wissenschafts- } \\
\text { propädeutische Aktivität }\end{array}$ & Forschung \\
\hline Fragestellung & $\begin{array}{l}\text { teilweise implizit und } \\
\text { teilweise explizit in Zielen und } \\
\text { Interessen } \\
\end{array}$ & $\begin{array}{l}\text { Explizites Formulieren, } \\
\text { Schriftlichkeit }\end{array}$ \\
\hline Hypothesenbildung & $\begin{array}{l}\text { Primäre und sekundäre } \\
\text { Erfahrung, Interesse }\end{array}$ & \begin{tabular}{|l|} 
Rekonstruktion des \\
Forschungsstands, \\
Forschungsinteresse \\
\end{tabular} \\
\hline Abstraktionsgrad & $\begin{array}{l}\text { Übung in abstrahierendem } \\
\text { Denken }\end{array}$ & Abstrakte Modelle \\
\hline Fachsprache & $\begin{array}{l}\text { Beginnende Differenzierung } \\
\text { von Sprachregistern je } \\
\text { Spielthema } \\
\end{array}$ & $\begin{array}{l}\text { Hoch elaborierte Nutzung von } \\
\text { Fachsprachen in Fachdiskursen }\end{array}$ \\
\hline Zeit & $\begin{array}{l}\text { kurze bis lange einzelne Spiel- } \\
\text { sequenzen sowie langfristige } \\
\text { Entwicklung und Differenzie- } \\
\text { rung in den Spielbereichen }\end{array}$ & $\begin{array}{l}\text { von Einzelergebnissen über } \\
\text { Projekte bis zu ganzen Paradig- } \\
\text { menwechseln im wissenschaft- } \\
\text { lichen Feld mehr oder weniger } \\
\text { zeitlich ausgedehnt }\end{array}$ \\
\hline Stand des Wissens & $\begin{array}{l}\text { Berücksichtigung des Vorwis- } \\
\text { sens anderer Spielender bzw. } \\
\text { der Spielgemeinschaft } \\
\end{array}$ & $\begin{array}{l}\text { Recherche des Forschungsstan- } \\
\text { des der scientific community }\end{array}$ \\
\hline Kategorie des Neuen & $\begin{array}{l}\text { (nach)entdeckende \& primäre } \\
\text { Erfahrungen } \rightarrow \text { Erkenntnisse } \\
\text { für sich selbst und die Spielge- } \\
\text { meinschaft }\end{array}$ & \begin{tabular}{|l|} 
Erweiterung des \\
Forschungsstandes
\end{tabular} \\
\hline Rolle von Regeln & schneller wechselbar & länger bindend \\
\hline
\end{tabular}

Den hier skizzierten Differenzen gegenüber sehen wir folgende Gemeinsamkeiten:

- Spiel und Forschung, bringen ein Interesse an Auseinandersetzung mit Wirklichkeit zum Ausdruck.

- Spiel und Forschung erproben und entwickeln Modelle von Wirklichkeit.

- Spiel und Forschung ist es wichtig, dass die Erfahrung von Wirklichkeit zur Darstellung, zum Ausdruck, zum Nachvollzug kommt.

- Spiel und Forschung sind mit einem „heiligen Ernst“ verknüpft.

- Spiel und Forschung wollen sozial ihren „Spielregeln“ Geltung verschaffen,

- Spiel und Forschung knüpfen an bestehendes gemeinschaftliches Wissen an und entwickeln es weiter, erlauben es, Neues zu erfahren.

Im Hinblick auf die Eingangsfragen nach dem kindlichen „Forschen“ und im Hinblick auf den Diskurs zu entdeckendem und forschendem Lernen in Lern- 
werkstätten und Forschungswerkstätten haben wir einerseits die Differenzen zwischen forschenden und entdeckenden Prozessen aufgezeigt. Über eine Betrachtung von Interaktions- und Konstruktionsprozessen im Spiel haben wir das dabei stattfindende gedankliche Konstruieren im Verhältnis zum forschenden Lernen neu beschrieben, und zwar nicht vor allem als einen Gegensatz, sondern in einem sich gegenseitig auf vielfache Weise fordernden Verhältnis. Daraus ergibt sich, dass spielerisches, entdeckendes und forschendes Lernen ihren Platz in Lern- und auch in Forschungswerkstätten haben sollten.

\section{Explorative vs. forschende Denkbewegungen von Kindern am Beispiel der „Lernwerkstatt Natur"}

Das nachfolgende Kapitel beleuchtet am Beispiel der „Lernwerkstatt Natur" ${ }^{\text {“6 }}$ in Mülheim an der Ruhr, wie junge Kinder zur „Sache“ kommen und welche Denkbewegungen dabei zentral sind. Aus diesen Überlegungen resultiert eine didaktische Frage: Wie können (früh)kindliche Lernprozesse unterstützt werden ohne dabei fachlich vorzeitig zu "disziplinieren“?

Im Rahmen des Projektes „Lernwerkstatt Natur“ haben Kindergartenkinder im Alter von zwei bis sechs Jahren die Möglichkeit, sich spielend, forschend und gestaltend mit Naturphänomenen auseinander zu setzen (Kleinow \& Panitz 2010: 108). Was meinen wir damit, dass sich Kinder in diesem Alter forschend mit etwas auseinandersetzen? Was tun sie tatsächlich? Welche Sprachen und Ausdrucksformen nutzen Kinder dabei? Inwieweit ähneln sie denen Erwachsener? Wer definiert, woran sich ihre Denkbewegungen orientieren oder zu orientieren haben? Welches Forschungs- und damit Lernverständnis legen wir zugrunde?

Wenn Kinder in Bildungseinrichtungen wie Kindergärten oder Schulen systematisiert Vorgegebenes erschließen sollen, ist es naheliegend, dass ErzieherInnen oder LehrerInnen ihnen dazu Werkzeuge bereitstellen, die diese Prozesse entlang der vorgedachten (didaktisierten) Wege ermöglichen. Wird die zu erfahrende Sache als „Forschungssache“ betrachtet, scheint der hinführende Prozess der des Forschens zu sein. Werden die Prozesse des Forschens an den wissenschaftlichen

5 Kapitel von: Kathleen Panitz

6 Forschungsprojekt der Universität zu Köln unter Leitung von Prof. Dr. Gerd E. Schäfer, in Trägerschaft der Stadt Mülheim an der Ruhr. Das Konzept der Lernwerkstatt Natur stützt sich auf praktische und theoretische Modelle von Dewey, Freinet und der Reggio-Pädagogik. Im Mittelpunkt der wissenschaftlichen Arbeit steht die Erforschung des kindlichen Denkens, insbesondere der Wege, auf denen sich Kinder die Welt erschließen, sowie die Entwicklung von Grundlagen einer auf diesen Erkenntnissen beruhenden Didaktik des Naturwissens. Das Projekt wurde durch die Deutsche Telekom Stiftung, das Ministerium für Generationen, Familien, Frauen und Integration des Landes Nordrhein-Westfalen und die Leonhard-Stinnes-Stiftung finanziert. 
Arbeitsweisen von ForscherInnen orientiert, lehnt sich der Diskurs an die Vorgehensweisen erwachsener Denkstrukturen an, die in Folge einer bestimmten Ausbildung und Expertise eine konkrete Fragestellung und Zielsetzung mit ausgewählten wissenschaftlichen Methoden zu einem eingegrenzten Sachverhalt verfolgen und die Ergebnisse ihrer Arbeiten publizieren und öffentlich zugänglich machen. Unter entwicklungspsychologischer Perspektive lässt sich verdeutlichen, dass sich formales und inhaltliches Denken bereits im Vorschulalter entwickelt und in der Schule formal-wissenschaftliches Denken mit einem wissenschaftstheoretischen Curriculum trainiert werden kann (vgl. Koerber 2006). Daraus ergibt sich ein scheinbareres (An)Passungsverhältnis der Denkweisen von jungen Kindern und Erwachsenen, womit sich Lernarrangements, z.B. mit Experimentiermöglichkeiten zur Lenkung kindlicher intuitiver Theorien und gezielten Förderung wissenschaftlicher Erkenntnisprozesse, stützen lassen. Es wird entwicklungslogisch deutlich, wozu Kinder bereits in jungem Alter fähig und kompetent im Sinne Dornes (1993) sind. Dennoch ergibt sich daraus auch der Blickwinkel des „noch-nicht-Könnens“ mit der Gefahr eines defizitär orientierten Blicks auf junge Kinder.

Schäfer (2017) kritisiert ein einseitig entwicklungspsychologisches Anpassungsverständnis als Grundlage für frühpädagogische Modelle. Er weist darauf hin, dass der wissenschaftliche Zugang ein gezielt selektives und diszipliniertes Denken erfordert und stellt in Frage, ob Kinder von vorn herein auf dieses methodisch eingeschränkte Denken vorbereitet werden sollen.

Wenn es weniger vordergründig darum gehen soll, zuvor festgelegte und disziplinär vorstrukturierte Inhalte nachzuvollziehen, stellt sich die Frage, wie sich junge Kinder die Welt und ihre Zusammenhänge erschließen. Wie lernen junge Kinder und welche Denkbewegungen spielen dabei eine Rolle? Es gilt genauer zu betrachten, inwieweit die eigenlogisch forschenden Bewegungen junger Kinder den sachlogischen Denk- und Handlungsweisen forschender Studierenden oder Wissenschaftler ähnlich sind und als Vorläufer dieser betrachtet werden können. Verwenden wir einen „kulturrelativistischen Blick“ (Schäfer 2017) auf die „Forschungssache“7, ändert sich auch die Perspektive auf die dahin führenden Prozesse. Das Verständnis frühkindlicher Bildungsprozesse ist durch die Kulturvorstellungen Erwachsener geprägt. Der kulturell strukturierte Blick wird vor allem durch die Schule vermittelt und sucht nach „Vorläufern“ in der frühen Kindheit, was sich bspw. an den Begriffen der „vorschulischen Bildung“ bzw. „Vorschule“ nachvollziehen lässt (vgl. ebd.). Interessanterweise besteht keine Notwendigkeit, die weiterführende Schule als „Voruniversität“ oder „Vorlehre“ bzw. die Berufstätigkeit als „Vorpension“ zu bezeichnen. Dass der institutionelle frühkindliche Bereich vordergründig aus der Perspektive der nachfolgenden Institu-

7 Die „Sache“, die forschend erschlossen werden soll und kulturell hervorgebracht wird. 
tion im Lebensabschnitt des Kindes betrachtet wird, schränkt andere Sicht- und Verständnisweisen ein. Dabei ist das Lernen vor und im Übergang in die Schule als „Denken mit ungeschultem Kopf“ (Gardner 2004 zitiert nach Schäfer 2017) zu betrachten. Schäfer (ebd.) plädiert deshalb dafür, dass Ansätze in der frühkindlichen Bildungsforschung benötigt werden, die die kulturelle Einteilung der Welt in Fächer und domänenspezifische Bereiche nicht unreflektiert voraussetzt. Diese Forderung kann auf die konkrete pädagogische Praxis in Lernwerkstätten, Kindergärten und in Anbetracht von Übergangs- und Transitionsprozessen in Primarschulen übertragen werden.

Schäfer (2013, 2014a, 2014b) stellt in seinen Arbeiten einen Lernbegriff in der Frühpädagogik in Frage, der von der Vermittlung vereinzelter Wissens- oder Kompetenzbereiche ausgeht. Stattdessen wird das Konzept des Erfahrungslernens zugrunde gelegt, dass das selbsttätige Lernen junger Kinder in Alltagszusammenhängen in den Vordergrund stellt und auf Handlungswissen der Kinder basiert. Kinder sind keine „kleinen Forscher“. Sie forschen nicht, sie explorieren (Schäfer 2017). Junge Kinder erkunden die Welt unsystematisch entlang ihren Erfahrungen. Sie bilden dabei aktives implizites Wissen. Sie nutzen dabei alle Werkzeuge und Materialien, die ihnen dazu zur Verfügung stehen, vordergründig ihre körperlichen, sensorischen und emotionalen Erfahrungsmöglichkeiten. Es sind alle Wahrnehmungs-, Handlungs- und Denkweisen zugelassen. Junge Kinder explorieren unmittelbar und vor allem in Erlebnis- und weniger in abstrakten Denkzusammenhängen ${ }^{8}$. So „klebt“ sich bspw. die 4-jährige Marie eine Schnecke, die sie unter einem Blatt entdeckt hat, an den Hals, um herauszufinden, ob sie sich auch an ihr „festhalten“ kann und stellt lachend fest: „Das kitzelt“. Das Mädchen, das ihr gegenübersteht, beobachtet genau was passiert. Auch sie hat ein Interesse an der Schnecke, allerdings ein anderes.

Das Erfahrungslernen unterscheidet sich somit in den Grundannahmen vom hochschuldidaktischen Konzept des forschenden Lernens und kann nicht ohne Weiteres auf junge Kinder übertragen werden. Auch wenn Kinder selbständig ihren impliziten Fragen und Hypothesen nachgehen, entwickeln sie diese nicht explizit. Sie formulieren kein Erkenntnisinteresse. Ihre Fragen ergeben sich unmittelbar, spontan und situativ. Sie benötigen keine Versuchsplanung, um ihren Erkenntnisinteressen nachgehen zu können. Sie untersuchen unsystematisch, gehen dabei nicht methodisch kontrolliert vor.

Junge Kinder nutzen vielfältige Methoden, vor allem die des eigenen Körpers und finden Antworten, die nicht zwangsläufig verbal sind. Beim Ausprobieren gibt es keine klare Abgrenzung zwischen Realität, Spiel, Fiktion und Phantasie. Auch

8 Die Formen des kindlichen Wissenszugangs werden mit den „Denkformaten“ des konkreten, aisthetischen, narrativen und theoretischen Denkens näher beschrieben (vgl. dazu ausführlich Schäfer 2014a: 293ff). 
wenn sie - insofern es nicht unterbunden wird - ihre Entdeckungsprozesse selbst gestalten, steht das Nach-sinnen nicht im Kontext eines expliziten Reflexionsprozesses. Ihre Erfahrungen sorgen für neue Fragen und Erkundungen und benötigen keine Aufbereitung und Präsentation für andere - wesentlicher sind die unmittelbaren konkreten und gestaltenden Ausdrucksformen.

Auch Plappert (2013: 4ff) gibt zu bedenken, dass sich Kinder und (Natur)Wissenschaftler in ihren Grundhaltungen unterscheiden. Auch wenn Kinder in der Lage sind, selbstgestellte Hypothesen zu überprüfen, haben sie kein Interesse an „wissenschaftlichen Erklärungen“ (ebd.). Forschen bedeutet für junge Kinder, sich selbst in Beziehung zu ihrer Umwelt zu setzen (Henneberg, Klein \& Vogt 2014: 146). Es geht nicht um das Inszenieren von Problemlöseprozessen zum Erreichen eines bestimmten Qualifikationsprofils an Wissen, Können und Selbstlernfähigkeiten, wie es mit dem Konzept des forschenden Lernens (Fichten 2010; Kottmann 2013) bspw. für die LehrerInnenbildung diskutiert wird. Kinder benötigen (zunächst) ein vorwissenschaftliches Erfahrungswissen, welches sie durch das Explorieren in Alltagszusammenhängen erlangen. „Dabei entwickeln sie eine Vielfalt von Denkweisen, die sie auch benötigen werden, um die Vielfalt wissenschaftlichen Denkens selbst zu entwickeln und zu begreifen" (Schäfer 2017).

Welche Konsequenzen ergeben sich aus diesen Überlegungen für die ErzieherInnen- und PrimarlehrerInnenbildung? Anstelle eines pädagogischen Verständnisses, das einseitig auf didaktische Rekonstruktion und Reduktion abzielt, sollten vielfältige Denkweisen eines breiten Zugangs angestrebt und in den Konsequenzen für die pädagogische Praxis diskutiert werden. Wir benötigen einen weiten und keinen einschränkenden Forschungsbegriff (Schäfer 2017). Auf dieser Grundlage ist Lehren und Lernen als Beziehungsgestaltung zwischen Kindern, Erwachsenen und Dingen im Sinne "gemeinsam geteilter Erfahrung“ in den Vordergrund zu stellen, bei der das Kind an den kulturellen Möglichkeiten seiner Umwelt beteiligt wird. Neben Beobachtung und Dokumentation als pädagogische Wahrnehmung und Verständigung („Partizipatorische Didaktik“ Schäfer \& von der Beek 2013), gilt es Wahrnehmung und Beobachtung als Grundlage eigener (auch biographisch bereits in der Vergangenheit liegender) Lern- und Bildungsprozesse der Studierenden in den Fokus zu rücken. Inwieweit ist es sinnvoll und notwendig, den Studierenden, ErzieherInnen und LehrerInnen selbst auch Exploration mit der Sache zu ermöglichen, anstatt es bei formal-wissenschaftlichen Sachanalysen in der Unterrichtsvorbereitung zu belassen, die sie womöglich mehr von den Denkweisen der Kinder entfernen, als ihnen diese näher zu bringen?

Demzufolge ist über eine Relativierung der Wissenshierarchie nachzudenken. Erwachsene wissen ggf. mehr über die Sache (z.B. die Schnecke) bzw. haben kulturelle Denkweisen dazu entwickelt (z.B. biologische oder kulinarische). Dies steht einem eher begrenzten Wissen über die individuellen Erkundungs- und Bildungsprozesse jedes einzelnen Kindes gegenüber. Eine Herausforderung für die Erzie- 
herInnen- und LehrerInnenbildung stellt somit das Erfahrbarmachen des biographischen und forschenden Lernens als kokonstruktiver Prozess (im Sinne Youniss 1994) zwischen situativer Neukonstruktion mit den Kindern und didaktischen Übertragungsmöglichkeiten eigener sachlicher Vorstellungen dar.

Dazu ein kleiner Exkurs anhand eines Beispiels aus der „Lernwerkstatt Natur“: Aus den Tätigkeiten einiger Kinder ergibt sich situativ die Idee und das gemeinsame Interesse der ErzieherInnen und Kinder, ein Floß zu bauen, mit dem die Kinder auf dem Teich im Wald schwimmen können. Betrachten wir diese Situation: Wie gehen Erwachsene vor, die mit 4- und 5-jährigen Kindern ein Floß bauen wollen, das anschließend schwimmen und ein Kind tragen soll?

Es werden Materialien gesichtet, implizite Hypothesen verfolgt und Ideen umgesetzt. Es kommen Fragen auf: Wie viele der vorrätigen leeren PET-Flaschen à 1,51 müssen unter dem aus Ästen zusammengesetzten ca. 10kg schweren Floß befestigt werden, um ausreichend Auftrieb zu erhalten? Ist das Konzept des Auftriebs das Richtige? Gibt es weitere Kräfte und Gesetzmäßigkeiten, die zu bedenken sind? Mitten im Wald sind Ideen und Lösungen gefragt, um das entstandene Projekt umsetzen zu können. Trotz erfolgreichem Schulabschluss und ggf. fächerübergreifendem problemorientierten Anwendungstraining probieren die Erwachsenen es „einfach“ aus, anstatt als erstes nach relevanten physikalischen Kenngrößen, Gesetzmäßigkeiten und Berechnungsmöglichkeiten zu suchen. Eine Mindestanzahl an PET-Flaschen wird geschätzt und befestigt, danach wird das Floß zu Wasser gelassen und peu à peu beschwert, um herauszufinden, wie viel es trägt. Auch wenn das Beispiel weder umfassend dargestellt noch analysiert ist, vergegenwärtigt es, dass (auch) Erwachsene explorativ vorgehen und erfahrungsbasiert in Alltagszusammenhängen lernen.

Abschließend lässt sich festhalten, dass Lernwerkstätten für junge Kinder keine Forschungswerkstätten sind, sondern Orte des Explorierens und Erfahrungslernens in Alltagskontexten. Kinder sind neugierig und interessiert, stellen Fragen, erkunden ihre Umwelt und machen dabei Entdeckungen. Es ist wichtig, ihre Denkbewegungen zu verstehen, diese in ihrer kindlichen Eigenlogik zu erhalten und zu unterstützen. Erwachsene sollten unbedingt weiter untersuchen und erforschen, wie Kinder „zur Sache“ kommen, dabei allerdings nicht in der eigenen Forschungslogik gefangen bleiben. 


\section{Forschen in Lernwerkstätten - Ein Plädoyer für eine didaktisch reflektierte Annäherung an ein dynamisches Lernfeld?}

„Reflektierendes Denken besteht in einem regen, andauernden und sorgfältigen Prüfen von etwas, das für wahr gehalten wird, und zwar im Lichte der Gründe auf die sich die Ansicht stützt, und der weiteren Schlüsse, denen sie zustrebt"

(Dewey 1910/1951: 6)

Das folgende Kapitel beschäftigt sich mit den Prozessen des Lernens und Lernbegleitens in Lernwerkstätten ${ }^{10}$. Auf Basis des VeLW Positionspapieres (VeLW 2009) wird zu skizzieren versucht, welche Besonderheiten ein auf Lernwerkstätten bezogenes Lernen (Ebene 1) und Lehren aufweist. Anhand ausgewählter Kriterien werden daraufhin korrespondierende Kompetenzen der Lernbegleitenden (Ebene 2) umrissen, um auf der Argumentationsfolie von Altrichter und Posch (2007) in Auseinandersetzung mit Schöns Reflexionsmodell (1983) der Frage nachzugehen, wie das Lernen in Lernwerkstätten unter seinen besonderen Bedingungen und Zielsetzungen adäquat forschungsmethodisch erfasst und analysiert werden kann. Aus dieser Auseinandersetzung sollen Überlegungen zu einem Kompetenzprofil für ein Forschen (Ebene 3) über Lernen in Lernwerkstätten angestoßen, zur Diskussion gestellt und anhand der Darstellung einer aktuellen empirischen Untersuchung in der Lernwerkstatt der Universität Kassel verdeutlicht werden.

Im Selbstverständnis von Lernwerkstätten und dem damit verbundenen Lernen in Lernwerkstätten besteht Übereinstimmung darüber, Lernen als einen offenen Prozess wahrzunehmen, der nicht nur einseitig auf Lernleistungserbringung zu einem definierten Lernziel reduziert werden kann, sondern die Gleichzeitigkeit der Lernebenen (kognitive wie affektive und psycho-motorische, lernstrategische und motivationale Lernebenen) in ihrer Gesamtheit berücksichtigt. Das Positionspapier des Verbundes europäischer Lernwerkstätten (VeLW 2009) rekurriert in diesem Sinne auf ein Lernverständnis als Qualitätsmerkmal von Lernwerkstattarbeit, das auf der Basis eines moderaten Konstruktivismus Lernen als aktiven und konstruktiven Prozess versteht. In diesem Verständnis rücken die Lernakteure in den Mittelpunkt, indem sie in ihren individuellen kumulativen Kompetenzentwicklungsprozessen eine „Neukonstruktion der Welt“ vornehmen (vgl. auch Dewey 1916/2011: 198) und dabei in situative Kontexten eingebunden sind, die einen sozialer Austausch ermöglicht (vgl. VeLW 2009: 6). Darüber hinausgehend enthält das Positionspapier weitere Qualitätsmerkmale, wie Fragen lernen, selbst-

9 Kapitel von: Maria Hummel und Ralf Schneider

10 Dies schließt ausdrücklich auch weitere Formen offener Lernarrangements u.a. in schulischen Settings mit ein. 
ständiges und selbstverantwortliches Arbeiten, individuelles und gemeinsames Arbeiten, Reflexion und Dokumentation des eigenen/gemeinsamen Lernprozesses, die das konstruktivistische Lernparadigma in eine operationalisierbare Form überführen (VeLW 2009: 7). Konsens besteht ebenfalls darin, der Heterogenität der Lernenden und ihrer Interessensunterschiede offen zu begegnen, ihre eigenen Fragestellungen, die Wahl der Methoden, die unterschiedlichen Zugänge zu den Gegenständen wertzuschätzen, anzunehmen und zu unterstützen sowie den sich entwickelnden Fragen Raum zu geben.

Diese Offenheit (Differenziert nach „Dimensionen“ bei Peschel 2016) und Selbstbestimmung der Lernenden ist für die Lernbegleitenden und ihre Moderationsfähigkeit dieser Lernprozesse eine besondere Herausforderung. Sie besteht, verkürzt gesagt, in der Unbestimmtheit zwischen Anfang und Ende: Zu Anfang steht zunächst eine unbestimmbare Kommunikations- und Interaktionssituation, es folgt eine unvorhersehbare Explorationsphase, der eine noch wagere, d.h. schwer zu antizipierende, Lernwegrealisation folgt. Ein Prozess, der mit ,Kontingenz' beschreibbar ist, sich aber schon in nascendi befindet, und sich erst im Vollzug realisiert. Diese Unbestimmtheit wird zunehmend dadurch bestimmbarer, dass sich u.a. Interessen, Fragen, Vermutungen etc. für die zu aufgenommenen Lernwege herauskristallisieren und von den Lernbegleitenden ko-konstruktiv erfasst werden. Die Ko-Konstuktion der Lernbegleitenden besteht in der Identifizierung sich abzeichnender Sinnfelder in einer Art Oszillationsbewegung, die in einem Prozess von Rekonstruktion und Antizipation erfasst werden und darauf aufbauend geeignete Impulse für ein erfolgreiches Lernen im Verlauf möglich machen. Das (vorläufige) Ende ist erreicht, wenn die Prozesse und Ergebnisse in einer Präsentationsphase kulminieren und reflexiv Lernwege und Lernergebnisse diskutiert und gewürdigt werden.

Eine solche Inszenierung des Lernens als dynamischer und mehrperspektivischer Prozess erfordert von den Lernbegleitenden umfassende didaktisch-methodische Kompetenzen für Planung, Begleitung, Dokumentation, Präsentation und Reflexion, die diese Unsicherheiten moderierend und reflektierend auszubalancieren verstehen.

Werden solche Handlungskompetenzen ${ }^{11}$ einer Lernbegleitung für komplexe Interaktionen zu Grunde gelegt, ist offensichtlich, dass sich offene Lernprozesse von Kindern nicht einfach einstellen oder durch Instruktion ermöglicht bzw. herbeigeführt werden können, sondern ein höchst komplexes und in seinen Folgen unsicher-fragiles Geschehen ist. Nach Schön (vgl. Schön 1983: 49, 276f) korrespondiert dieses anspruchsvolle didaktische Design mit der Figur eines Professionals,

11 Handlungskompetenz in dynamischen Interaktionen umfassen dabei fachliches, fach- und allgemeindidaktisches, methodisches, lernpsychologisch-motivationales, lerngegenstandsbezogenes Wissen. 
die/der im professionellen Handeln zur Evaluation der Qualität des Lehr-/Lernprozesses über ein dreistufiges Reflexionsverfahren disponiert und entsprechend weiteres Handeln neu justiert. Der Rückgriff auf eine der drei Reflexionsebenen (implizit knowing in action, reflection in action, reflection on action) steht dabei in Abhängigkeit von den jeweiligen situativen Bedingungen, den Prozessverläufen und der Komplexität der darin auftauchenden Probleme (vgl. Altrichter \& Posch 2007: 320ff).

Auf der Folie dieses Entwurfs eines professionellen Handlungsmodells, das in der Figur eines Reflective Practitioners firmiert, gehen Altrichter \& Posch (2007: 329ff) der Frage nach, ob und wie diese Kompetenzen für die Weiterentwicklung zu einem forschenden Habitus, insbesondere in Settings für die sich ein Aktionsforschungsansatz anbietet, genutzt werden könnten. Dabei gehe es darum, dass in diesen Forschungsprozessen -und das trifft für das offene Lernen in Lernwerkstätten $\mathrm{zu}$ - allgemein- und fachdidaktisches, methodisches, fachliches Wissen etc. als Prozesswissen benötigt wird und für die Entwicklung eines Forschungsdesigns bedeutsame Impulse setzen könnte. Das läuft letztlich auf die Vorstellung hinaus, dass sich damit komplexes Handlungs- und Reflexionswissen, erweitert um forschungsmethodisches Know-how, zu einer Figur eines Lehrer-Forschers zusammenfügen könnte.

Interessant an dieser Figur des Lehrer-Forschers erscheint uns nicht so sehr das Verhältnis zwischen den beiden Wissenstypen des praktischen Handlungswissens und des wissenschaftlichen Wissens genauer auszuloten, auch nicht die Frage zu beantworten, wie der Übergang von der Schulpraxis zur Wissenschaft zu bewältigen ist. Wir sehen im Spiegel der Diskussion über die Kompetenzen eines des Reflective Practitioners eher Hinweise auf die besonderen Voraussetzungen über die ForscherInnen verfügen müssen, um anspruchsvolle Forschungsdesigns für Lernsettings in Lernwerkstätten anzulegen. In Anlehnung an das Kompetenzprofil von Reflective Practitioners sollten Forschende über eine hohe Sensibilität für die besondere Qualität der arrangierten offenen und lernendenzentrierten Lernprozesse zwischen dem dargestellten Anfang und Ende, zwischen Aufgabe und Reflexion sein.

Diese Überlegungen führen zu einem, wenngleich nicht vollständigen, Kompetenzprofil und einem Erwartungshorizont für das Forschen in Lernwerkstätten und setzt folgende Kenntnisse voraus:

- Erkennen zu können, welcher Zusammenhang zwischen Aufgabenformat, Aufgabenstellung (auch selbstgestellte) zu Anfang, Eigenschaften des Materials und Hilfe bei der Auswahl, Erarbeiten/Experimentieren/Entdecken und begleiten und moderieren der Prozesse und einer darauf basierenden Reflexion besteht.

- Einzuschätzen, welche Aufgaben für welche Kinder lernwirksam sind und mit welchen Impulsen Unterstützung im Sinne eines Scaffolding geleistet werden 
können bzw. wahrzunehmen, welche je eigenen Auseinandersetzungen möglicherweise verhindert werden.

- Erfassen zu können, in welchen Konstellationen kooperative Lernphasen für wen besonders lernunterstützend sein können, und wie diese kooperativen Phasen moderiert bzw. sinnvoll gesteuert werden können.

- Abwägen zu können, welche Bedeutung die Struktur und Zugänglichkeit von Lernmaterialien haben und alternative lernförderliche Materialien erkennen können.

- Ermessen, wie Lernende aktiviert werden können und mit welchen Fragen selbstgesteuerte Lernprozesse unterstützt werden können.

- Abschätzen zu können, mit welchen unterschiedlichen Methoden Kinder einen Gegenstand untersuchen/bearbeiten, und welche Folgen das für den Entdeckungsprozess hat.

- Erwägen zu können, über was die Reflexion Auskunft geben soll, wovon sie abhängig ist und wie diese Phase angelegt werden kann, um die Ergebnisse lernwirksam zu sichern.

Ein Beispiel: Deutsch als Zweitsprache (DaZ) in der Lernwerkstatt Kassel: (Sprach-)Lernprozesse zwischen Handlungs- und Forschungsperspektive

Im Rahmen des Projektseminars „Passungen finden“, das in Kooperation mit einer Kasseler Grundschule in der Grundschulwerkstatt der Universität Kassel durchgeführt wird, setzen sich Lehramtsstudierende mit der Frage auseinander, wie sich entdeckende Lernumgebungen für alters- und herkunftsmäßig heterogene Lerngruppen so gestalten lassen, dass Lernpotenziale der Kinder erkannt und entwickelt werden können. Innerhalb dieser umfassenden Zielsetzung wird ein Forschungsprojekt realisiert, das sich mit Spracherwerbsprozessen dieser Kinder mit Deutsch als Zweitsprache auseinandersetzt. Die Studie untersucht, welche Bedingungen im Lernsetting sich als günstig für den Erwerb bildungssprachlicher Kompetenzen der Kinder herausstellen. Die Idee für das Forschungsvorhaben entwickelte sich aus ForscherInnenperspektive aus der teilnehmenden Beobachtung von Sprachentwicklungsprozessen in offenen Projektsettings und der Begleitung mehrerer Projektphasen. Die Projektangebote (z.B. Statik, Nähen, Entstehung von Filmen, Lebensräume von Tieren, Holzwerkstatt) stellten sich durch den außerunterrichtlichen Charakter des Lernens unter den Bedingungen einer freiwilligen Teilnahme als besonders günstig für das Anbahnen von Sprachlernprozessen (u.a. durch beschreibendes, begleitendes, befragendes, argumentierendes Sprachhandeln) heraus. Darüber hinaus wiesen die Projekte Merkmale auf, die unter lernmotivationalen Gesichtspunkten erfolgsversprechend waren, da sie insbesondere den von Deci und Ryan (1993: 229) formulierten Grundbedürfnissen von Lernenden nach Selbstwirksamkeit, Selbstbestimmung und sozialer Eingebundenheit gerecht werden (vgl. Haenisch 2011: 10) und günstige Bedingungen für individuelle Sprachlernprozesse boten. Durch kooperative Arbeit in kleineren 
Projektgruppen ergaben sich für Kinder zahlreiche Möglichkeiten zum aktiven Sprachhandeln; durch Handlungsorientierung und herausfordernde Aufgaben eröffnen sich für sie Zugänge zur Sprache, durch fehlenden Leistungsdruck und Orientierung an ihren eigenen Interessen entstand ein geschützter Raum zum Ausprobieren und Experimentieren mit den Dingen, wie mit der Sprache (fragen, erklären, bitten, darstellen, beschreiben, vergleichen, vermuten etc.).

Diese zunächst nur begleitenden Beobachtungen führten zu ersten Überlegungen darüber, mit welchem Untersuchungsdesign der Zusammenhang zwischen offenen Lernsituationen und Sprachaktivitäten am besten zu ermitteln sind: Welche Fragestellungen sind im vorgefundenen Untersuchungskontext sinnvoll? Welche Forschungsmethoden erscheinen dem Gegenstand angemessen? Dabei haben sich als leitende Forschungsfragen folgende Aspekte herauskristallisiert:

- In welchen Situationen/Phasen des Lernens in offenen Lernumgebungen ist die Konzeptbildung der Kinder auf die Sprache angewiesen?

- Welche Diskursfunktionen (beschreiben, erklären, begründen, bewerten etc.) lassen sich in den eruierten Situationen/Phasen identifizieren?

- Wie werden die identifizierten Diskursfunktionen von Kindern sprachlich realisiert? Lassen sich hierbei bestimmte Formulierungsmuster erkennen?

Als Datenerhebungsmethode wurde die audio- bzw. videogestützte nicht-teilnehmende Beobachtung festgelegt; die so erhobenen Daten sollten diskursanalytisch ausgewertet werden. Im Verlauf der ersten Untersuchungsphase wurde schnell deutlich, dass die vordefinierten Beobachtung- und Analysekategorien das Seminargeschehen nur unzureichend erfassen konnten. Sie sind der Dynamik der stattfindenden Prozesse nicht gerecht geworden, da das Sprachverhalten der Kinder von zahlreichen Variablen abhängt, die sich einerseits auf themenimmanente Inhalte und Arbeitsweisen, andererseits auf didaktisch-methodische Entscheidungen der die Kinder begleitenden Studierenden zurückführen lassen.

Da die Forscherin nicht nur das Handeln der Studierenden bei der Begleitung der Kinder beobachtete, sondern auch in Seminarphasen anwesend war, in denen für das Lernen in Lernwerkstätten relevante Konzepte der Anbahnung und Begleitung von Entdeckungsprozessen der Kinder diskutiert und in Beziehung zu konkreten gemeinsam mit Kindern erlebten Situationen gesetzt wurden, registrierte sie einen Wandel im eigenen Denkparadigma. Sie musste feststellen, dass das Sprachverhalten der Kinder nicht isoliert von solchen Aspekten wie Aufgabenstellung, Zugänglichkeit des Materials, Strukturierung der Lernumgebung, kognitive Aktivierung der Kinder, Repräsentationsebenen, auf denen sich die Kinder befinden, etc. erforscht werden kann und dass ein Untersuchungsdesign, das die für den Zugewinn an bildungssprachlichen Kompetenzen förderlichen Bedingungen ermittelt, alle genannten Aspekte berücksichtigen sollte. 
Besonders deutlich werden in den Darstellungen des Lernwerkstattprojektes und einer entsprechend angelegten Forschung über das Lernen in Lernwerkstätten unter einer spezifisch linguistischen Perspektive u.a. folgende Aspekte:

Die Dynamik der Lehr-Lernprozesse, die besonderen Grade an Offenheit gegenüber den individuellen Entdeckungen der Lernenden, die nicht immer und zu jeder Zeit vollständig zu bestimmen sind, bilden auch für Forschende spannende Einblicke in ein kreatives Lernmilieu.

Die simultanen und sich oft ungleichzeitig vollziehenden Entwicklungs- und Entdeckungsprozesse nötigen den Forschenden ein breitgefächertes Wissen ab (u.a. didaktisches, fachdidaktisches, fachliches, methodisches, lernpsychologisches, aufgabenentwickelndes Wissen).

Das Finden eines passenden Forschungsdesigns ist in erheblichem Maße von einem engagierten Dabei- und Dazwischen-Sein, also in einer Art reflexiven Mitpräsenz der Forschenden in der Rolle als didaktisch versierte teilnehmende BeobachterInnen, abhängig.

Damit verknüpft ist notwendigerweise eine didaktische, kontextbezogene und reflexive Sensibilität bei der Datenanalyse und -interpretation.

Die Rolle des Lernbegleiters bestimmt sich in diesem Setting von der Moderation komplexer Lernprozessen her, deren Anfang unbestimmt und deren Ausgang unsicher ist, mit der Intention erfolgreiches Lernen mehrdimensional zu initiieren. Die Rolle der Forschenden bestimmt sich in diesem Spannungsfeld wenig kontrollierbarer und bestimmbarer Variablen zusätzlich durch das Ziel, Kriterien für ein erfolgreiches Lernen zu lokalisieren. Das Miterleben unterschiedlicher Aneignungs-, Darstellungs- und Repräsentationsformen in einem offenen Entwicklungsprozess erfordert von Forschenden eine kontextsensible und induktive, wenn man so will ethnografische Erkenntnishaltung, die aus dem Mitvollzug des beabsichtigen/unbeabsichtigten Lerngeschehens eine Begleitforschung generiert, in der es vorrangig um das Aufspüren der Vernetzung kindlicher Erfahrung, entsprechender Motivationslage, der Dynamisierung affektiven, psychomotorischen und kognitiven Lernens geht.

Wie gezeigt wurde, fallen diese genannten Kompetenzen in der Regel in das Aufgabenprofil des Reflective Practitioners als InitiatorIn und ModeratorIn von Lernprozessen und nicht in das des/der Forschenden. Aber: Nach unseren voranstehenden Darstellungen und Argumentation steht diese Trennung für das Forschen in Lernwerkstätten zur Disposition. Der Anspruch, die besonderen Potenziale von Lernwerkstätten zu untersuchen, setzt eine besondere und lernprozessaffine Optik voraus, die sich u.E. nur entfaltet in der Figur des Reflective Researchers. 


\section{Literatur}

Altrichter, Herbert \& Posch, Peter (2007): Lehrer erforschen ihren Unterricht. Eine Einführung in die Methoden der Aktionsforschung. Klinkhardt Verlag: Bad Heilbrunn, 4. Aufl.

Andresen, Helga (2002): Interaktion, Sprache und Spiel: zur Funktion des Rollenspiels für die Sprachentwicklung im Vorschulalter. Gunter Narr Verlag: Tübingen.

Andresen, Helga (2003): Zur Bedeutung des Rollenspiels für die Sprachentwicklung im Vorschulalter. In: Panagiotopoulou, Argyro und Brügelmann, Hans (Hrsg.): Grundschulpädagogik meets Kindheitsforschung. Bd. 7. VS Verlag für Sozialwissenschaften: Wiesbaden. 135-139.

Andresen, Helga (2011): Erzählen und Rollenspiel von Kindern zwischen drei und sechs Jahren: eine Expertise der Weiterbildungsinitiative Frühpädagogische Fachkräfte (WiFF). DJI: München.

Angelone, Domenico, Keller, Florian \& Moser, Urs (2013). Entwicklung schulischer Leistungen während der obligatorischen Schulzeit. Zürich: Bildungsdirektion Kanton Zürich.

Beywl, Wolfgang, Künzli, D., Künzli, Christine, Messmer, Roland \& Streit, Christine (2015). Forschungsverständnis pädagogischer Hochschulen. Ein Diskussionsbeitrag. Beiträge zur Lehrerinnen- und Lehrerbildung, 33(1), 134-151.

Bose, Ines (2003): dóch da sín ja’ nur mûster II: Kindlicher Sprechausdruck im sozialen Rollenspiel. Peter Lang: Frankfurt am Main; New York.

Buck, P. (2012): Verstehen kann nur jeder für sich selbst (Wagenschein). Wie wird aus einem Phänomen vor mir ein wissenschaftlicher Begriff in mir? In: Kruse, N. u.a. (Hrsg.) (2012): Martin Wagenschein - Faszination und Aktualität des Genetischen. Schneider Verlag Hohengehren: Baltmannsweiler, 83-99.

Deci, Edward L. \& Ryan, Richard M.: Die Selbstbestimmungstheorie der Motivation und ihre Bedeutung für die Pädagogik, in: Zeitschrift für Pädagogik, 1993, 39/2, S. 223-238.

Dewey, John (1910/1951): Wie wir denken. Eine Untersuchung über die Beziehung des reflektiven Denkens zum Prozeß der Erziehung. Morgarten Verlag Conzett \& Huber, Zürich, S. 6.

Dewey, John (1916/2011). Demokratie und Erziehung. Eine Einleitung in die philosophische Pädagogik. Herausgegeben mit einem Nachwort von Jürgen Oelkers, Beltz Verleg, Weinheim und Basel, 5. Aufl., S. 198.

Dornes, Martin (1993): Der kompetente Säugling: Die präverbale Entwicklung des Menschen. Frankfurt am Main: Fischer.

Fichten, Wolfgang (2010): Forschendes Lernen in der Lehrerbildung. In: Eberhardt, U. (Hrsg.): Neue Impulse in der Hochschuldidaktik. Heidelberg: VS-Verlag, 127-182.

Franz, E.-K. (2012): Lernwerkstätten an Hochschulen. Orte der gemeinsamen Qualifikation von Studierenden, pädagogischen Fachkräften des Elementarbereichs und Lehrkräften der Primarstufe. Peter Lang Verlag: Frankfurt a. M.

Giffin, Holly (1984): The Coordination of Meaning in the Creation of a Shared Make-believe Reality. In: Bretherton, Inge (Hrsg.): Symbolic Play. Academic Press. S. 73-100.

Gruhn, A. (2017 i.E.): „Ist das, was wir hier machen, eigentlich Lernen?“ - Überlegungen zum Lernen in Hochschullernwerkstätten. In: Kahnwald, Nina u.a. (Hrsg.): Informelles Lernen. Eine Standortbestimmung. Springer VS: Wiesbaden.

Gruhn, A. \& Müller-Naendrup, B. (2014): „We don't need no education!“ - Hochschullernwerkstätten zwischen Angebot und Nachfrage. In: Hildebrandt, E. u.a (Hrsg.): Lernen zwischen freiem und instruiertem Tätigsein. Klinkhardt Verlag: Bad Heilbrunn, 146-159.

Gruhn, A. \& Müller-Naendrup, B. (2016, in Druck): „Theoretische Kreativität“ in Hochschullernwerkstätten - ein Plädoyer. In: Kekeritz, M. u.a. (Hrsg.): Lernwerkstatt als Prinzip. Möglichkeiten für Lehre und Forschung. Klinkhardt Verlag: Bad Heilbrunn.

Haenisch, Hans: Empirische Befunde über Aktivitäten und Rahmenbedingungen von Sprachbildung in offenen Ganztagsschulen, in: Hein\& Prinz-Wittner, Beim Wort genommen! Chancen integra- 
tiver Sprachbildung im Ganztag, Heft 20 der Reihe „Der GanzTag in NRW“ der Serviceagentur „Ganztägig lernen in Nordrhein-Westfalen“, Münster, 2011, S. 10-15.

Hagstedt, H. (2014): Unterrichtsentwicklung braucht anspruchsvolle Lernumgebungen. In: Hildebrandt, E. u.a. (Hrsg.): Lernen zwischen freiem und instruiertem Tätigsein. Klinkhardt Verlag: Bad Heilbrunn, 123-136.

Hagstedt, Herbert und Krauth, Ilse Marie (2014): Lernwerkstätten: Potenziale für Schulen von morgen. Grundschulverband - Arbeitskreis Grundschule: Frankfurt am Main.

Hauser, Bernhard (2013). Spielen: Frühes Lernen in Familie, Krippe und Kindergarten. Stuttgart: W. Kohlhammer.

Heimlich, Ulrich (2015): Einführung in die Spielpädagogik. 3 Aufl. Klinkhardt: Bad Heilbrunn.

Henneberg, Rosy; Klein, Lothar \&Vogt, Herbert (2014): Das Wunderbare der Entdeckungen. Kindertageseinrichtungen als Lernwerkstätten. In: Hagstedt, Herbert \& Krauth, Ilse Marie (Hrsg.): Lernwerkstätten. Potentiale für Schulen von morgen. Frankfurt am Main: Grundschulverband, 139-150.

Hildebrandt, Elke; Güvenç, Ezgi und Pautasso, Johanna (2015): Begleitung von Rollenspiel im Kindergarten. In: Lernprozessbegleitung und adaptives Lernen in der Grundschule. Springer VS: Wiesbaden, 253-258.

Hildebrandt, Elke; Güvenç, Ezgi und Pautasso, Johanna (2016): Das Rollenspiel als inkludierende Spielform - Erkundungen zur Verwendung von Sprache im Rollenspiel. In: Schmude, Corinna und Wedekind, Hartmut (Hrsg.): Lernwerkstätten an Hochschulen: Orte einer inklusiven Pädagogik. Klinkhardt: Bad Heilbrunn, 188-200.

Hildebrandt, Elke; Nieswandt, Martina; Radtke, Monika; Schneider, Ralph und Wildt, Johannes (2014): Werkstätten als Raum für Forschendes Lernen in der Hochschulbildung. In: Hildebrandt, Elke; Peschel, Markus; und Weißhaupt, Mark (Hrsg.): Lernen zwischen freiem und instruiertem Tätigsein. Klinkhardt: Bad Heilbrunn, 80-99.

Hildebrandt, Elke; Weisshaupt, Mark (2018 i.V.): Vom Spielbewusstsein zum Forschenden Lernen. In: Tagungsband zur Tagung Forschendes Lernen - The Wider View, in Münster vom 25.-27. September 2017, TM-Verlag

Huber, Ludwig; Hellmer, Julia und Schneider, Friederike (2009): Forschendes Lernen im Studium. Aktuelle Konzepte und Erfahrungen. UVW: Bielefeld.

Huizinga, Johan (2011): Homo Ludens: Vom Ursprung der Kultur im Spiel. 22 Aufl. Rowohlt Taschenbuch: Reinbek.

Humboldt, Wilhelm von (1809): Über die innere und äußere Organisation der höheren wissenschaftlichen Anstalten in Berlin. In: Königlich-Preußische Akademie der Wissenschaften (1934), 250260.

Jörissen, Benjamin (2009): Stichwort: „Spiel und Bildung“. In: DIE Zeitschrift für Erwachsenenbildung. Heft 3/2009, 24-25.

Kleinow, Matthias \& Panitz, Kathleen (2010): Lernwerkstatt Natur. Erfahrungslernen als Grundlage von Naturwissen. In: KiTa aktuell. 19. Jg., 5/2010, 108-110.

Koerber, Susanne (2006): Entwicklung des wissenschaftlichen Denkens bei Vier- bis Achtjährigen. In: Beiträge zur Lehrerbildung, 24/ 2, 192-201.

Kottmann, B. (2013): Forschendes Lernen in Lernwerkstätten. In: Coelen, H. u.a.: Studieren in Lernwerkstätten. Springer Fachmedien Wiesbaden: Wiesbaden, 183-191.

Kottmann, Brigitte (2013): Forschendes Lernen in Lernwerkstätten. In: Coelen, Hendrik und MüllerNaendrup, Barbara (Hrsg.): Studieren in Lernwerkstätten. Springer Fachmedien: Wiesbaden. 183191.

Kottmann, Brigitte (2013): Forschendes Lernen in Lernwerkstätten. In: Coelen, Hendrik \& Müller-Naendrup, Barbara (Hrsg.): Studieren in Lernwerkstätten. Wiesbaden: Springer Fachmedien 
Wiesbaden, 183-191, http://link.springer.com/10.1007/978-3-658-00315-9_15 [letzter Zugriff am 27.09.16].

Kucharz, Diemut; Mackowiak, Katja; Ziroli, Sergio; Kauertz, Alexander; Rathgeb-Schnierer, Elisabeth und Dieck, Margarete (2014): Professionelles Handeln im Elementarbereich (PRIMEL): Eine deutsch-schweizerische Videostudie. Münster New York: Waxmann.

Morris, Bradley; Croker, Steve; Mansick, Amy und Zimmerman, Corinne (2012): The Emergence of Scientific Reasoning. In: Kloos, Heidi (Hrsg.): Current Topics in Children's Learning and Cognition. InTech. [http://www.intechopen.com/books/current-topics-in-children-s-learning-andcognition/the-emergence-of-scientific-reasoning; 4.10.2016].

Müller-Naendrup, B. (2012): Lernwerkstätten an Hochschulen. Räumliche Botschaften im Rahmen der Lehrerbildung. In: Schrödeler v. Brandt, H. u.a.: Raum für Bildung. Ästhetik und Architektur von Lern- und Lebensorten. transcript Verlag: Bielefeld, 275-284.

Müller-Naendrup, B. (2015): Wenn Grundschulkinder und Studierende mit Muße lernen. Erfahrungen aus der Siegener Lernwerkstatt OASE. In: Pädagogik 10/2015, 30-33.

Oelkers, Jürgen (1997): How to define and justify scientific literacy for everyone. In: Scientific Literacy: An International Symposium. Kiel: IPN. [http://www.ife.uzh.ch/research/emeriti/oelkersjuergen/vortraegeprofoelkers/englishlectures/ScientificLiteracy.pdf; 26.7.2017].

Oerter, Rolf (1999). Psychologie des Spiels: Ein handlungstheoretischer Ansatz (Durchges. Neuausgabe). Weinheim: Beltz.

Peschel, Falko (2016): Offener Unterricht - Idee, Realität, Perspektive und ein praxiserprobtes Konzept zur Diskussion. Teil I: Allgemeindidaktische Überlegungen. Schneider Verlag, Hohengehren, 7. Aufl.

Peschl, M. F. \& Fundneider, T. (2010): Emergente Innovation. Wie es möglich wird, in Enabling Spaces das radikal Neue hervorzubringen. In: Pircher, R. (Hrsg.): Wissensmanagement. Wissenstransfer. Wissensnetzwerke. Publicis Publishing: Erlangen, 264-279.

Plappert, Dieter (2013): Naturkundliche und naturwissenschaftliche Bildung von der frühen Kindheit bis ins Erwachsenenalter als Ganzes betrachtet, http://www.oekostation.de/docs/Leitlinien_Naturkundliche_Bildung_Plappert_2013_final_75.pdf [letzter Zugriff am 20.12.2017].

Popper, Karl R. (2009): Vermutungen und Widerlegungen: das Wachstum der wissenschaftlichen Erkenntnis. Mohr Siebeck: Tübingen.

Schäfer, Gerd E. (2013): Der Bildungsbegriff in der Pädagogik der frühen Kindheit. In: Fried, Lilian \& Roux, Susanna (Hrsg.): Handbuch Pädagogik der frühen Kindheit. Berlin: Cornelsen, 33-44.

Schäfer, Gerd E. (2014a): Was ist frühkindliche Bildung? Kindlicher Anfängergeist in einer Kultur des Lernens. 2. Auflage. Weinheim und Basel: Beltz.

Schäfer, Gerd E. (2014b): Partizipatorische Didaktik in der Lernwerkstatt Natur. In: Hagstedt, Herbert $\&$ Krauth, Ilse Marie (Hrsg.). Lernwerkstätten. Potentiale für Schulen von morgen. Frankfurt am Main: Grundschulverband, 122-138.

Schäfer, Gerd E. (2017): Denkweisen des Naturwissens. Um welche Naturwissenschaft geht es? In: Mathis, Christian \& Favre, Pascal (Hrsg.): Naturphänomene verstehen. Baltmannsweiler: Schneider Verlag Hohengehren, 29-40.

Schäfer, Gerd E. \& von der Beek, Angelika (2013): Didaktik in der frühen Kindheit. Von Reggio lernen und weiterdenken. Berlin: Verlag das Netz.

Schäfer, Gerd E. und Hauser, Jutta (2014): „Aus dem Handeln heraus begreifen...“ Das pädagogische Interesse am Erfahrungslernen wächst. In: Theorie und Praxis der Sozialpädagogik, Heft 7, 8-11.

Schneider, Ralf und Wildt, Johannes (2002): Forschendes Lernen in Praxisstudien. Das Beispiel des Berufspraktischen Halbjahres in der Lehrerausbildung. In: B. Berendt u.a. (Hrsg.), Neues Handbuch Hochschullehre, Teil G 3.1: Schlüsselqualifikationen und wissenschaftliches Arbeiten. Berlin: Raabe. 
Schneider, Ralf (2009): Kompetenzentwicklung durch forschendes Lernen. In: journal hochschuldidaktik, Heft 20/2, 33-37.

Schneider, Ralph und Schwarzkopf, Theresa (2013): Wie viel Lernwerkstatt steckt in einer Forschungswerkstatt? In: Coelen, Hendrik und Müller-Naendrup, Barbara (Hrsg.): Studieren in Lernwerkstätten. Springer Fachmedien: Wiesbaden, 171-181.

Schön, Donald A. (1983): Reflective Practitioner: How Professionals Think In Action. Basic Books, USA.

Verbund europäischer Lernwerkstätten (VeLW) e.V. (Hrsg.) (2009): Positionspapier des Verbundes europäischer Lernwerkstätten (VeLW) e.V. zu Qualitätsmerkmalen von Lernwerkstätten und Lernwerkstattarbeit. Bad Urach.

Wedekind, Hartmut und Schmude, Corinna (2017): Werkstätten an Hochschulen - Orte des entdeckenden und/oder forschenden Lernens, In: Kekeritz, Monika u.a. (Hrsg.): Lernwerkstattarbeit als Prinzip. Klinkhardt: Bad Heilbrunn, 185-200.

Weisshaupt, Mark und Campana, Sabine (2014): Spielbewusstsein und Bildung beim sozialen Spiel. In: Hildebrandt, Elke; Peschel, Markus; und Weißhaupt, Mark (Hrsg.): Lernen zwischen freiem und instruiertem Tätigsein. Klinkhardt: Bad Heilbrunn, 43-66.

Wygotski, Lew S. (1980): Das Spiel und seine Bedeutung in der psychischen Entwicklung des Kindes. In: Elkonin, Daniil (Hrsg.): Psychologie des Spiels. Lehmanns Media: Berlin, 441-465.

Youniss, James (1994): Soziale Konstruktion und psychische Entwicklung. Frankfurt: Suhrkamp. 